\title{
Part 3
}

\section{Extra-Galactic Star Formation}




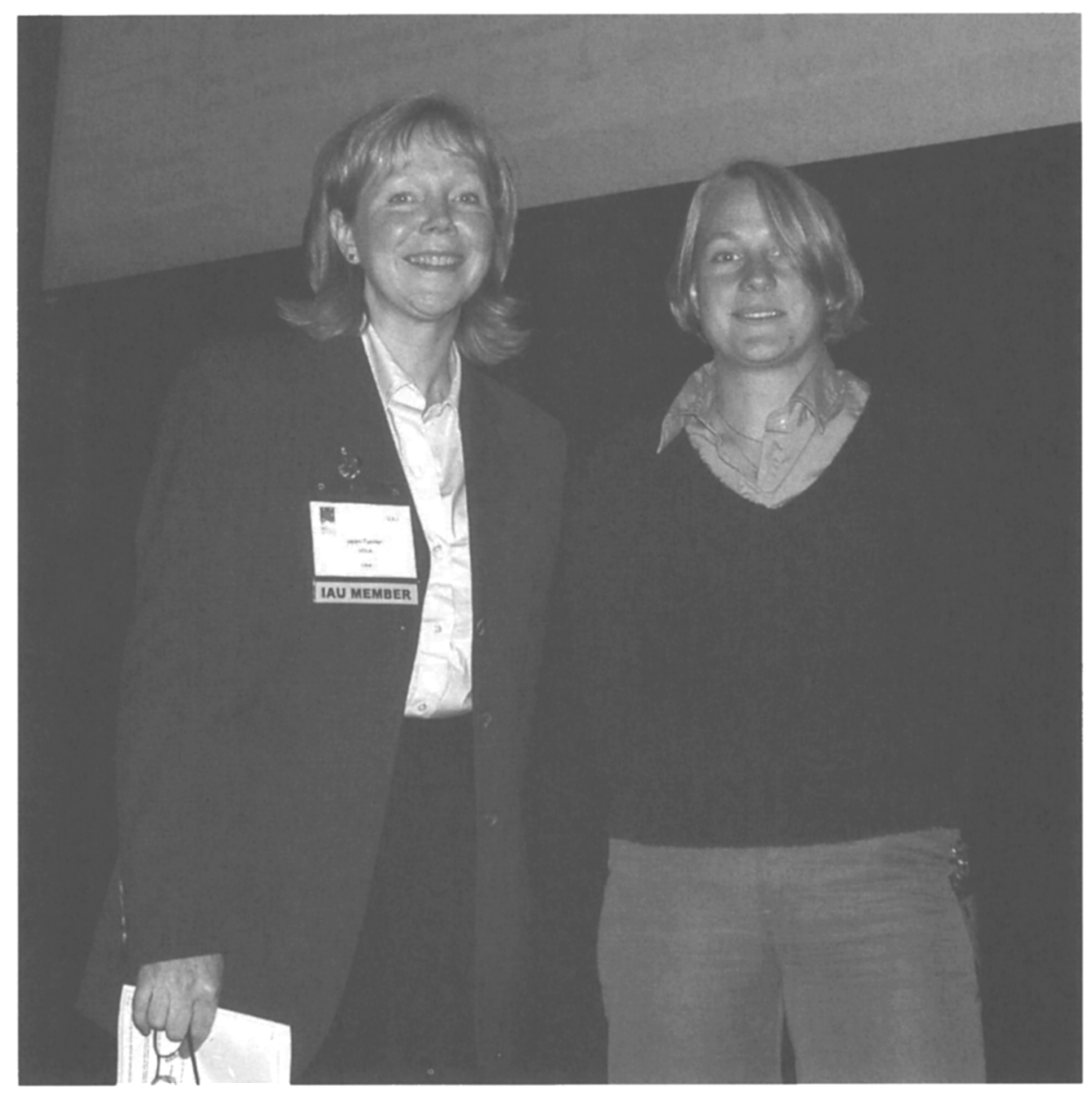

Jean Turner and Eva Schinnerer 


\title{
Extra-galactic Star Formation Revealed
}

\author{
Eva Schinnerer \\ National Radio Astronomy Observatory \\ 1003 Lopezville Road \\ Socorro, NM 87801, U.S.A.
}

\begin{abstract}
High angular resolution observations of nearby galaxies in the optical using ground-based and space-based telescopes have not only revealed the presence of young stellar clusters, but also allowed to study their properties in various dynamical environments. These studies have shown that young massive clusters (YMCs) have typical masses of a few $1000 \mathrm{M}_{\odot}$ and sizes of a few parsec irrespective of their site of formation (such as bulges, spiral arms, starburst rings, or mergers). This points toward a universal formation mechanism for these stellar clusters.
\end{abstract}

Observations of the dust and gas content in high redshift galaxies allows one to study the reservoir for star formation in the early universe. These studies reveal extremely high star formation rates of a few 1000 $\mathrm{M}_{\odot} \mathrm{yr}^{-1}$, while the distribution of the molecular gas still seems to be comparable to what is observed in the local universe. The detection of considerable amounts of molecular gas via its CO lines in the highest redshifted QSOs known today (up to $\mathrm{z}=6.4$ ) indicates that star formation in the early universe has already produced considerable amounts of metals.

\section{Introduction}

High angular resolution observations have resulted in tremendous progress in the understanding of star formation processes in external galaxies in recent years. Studies of nearby galaxies reveal important insights into the overall star formation processes, such as the preferred environments where new stars are formed, the mechanisms that might govern their formation, as well as the mode or history of star formation. By extending this work to galaxies seen at high redshifts, it is now possible to probe the star formation history of the early universe. These studies are essential to investigate such important topics as the universality of the star formation process over the age of the universe, and the cause for the apparent higher star formation rates seen in the young universe.

Compared to Galactic star formation, observations of extragalactic star formation are strongly biased to the formation of high-mass stars due to sensitivity limitations. Usually these high-mass stars are observed to form in stellar clusters. One key issue is to investigate the impact of different triggering mechanisms onto the star formation modes in nearby galaxies. The following trigger mechanisms have been found to play an important role: (a) random: localized 
sites of a few stellar clusters (e.g. as seen in dwarf irregular galaxies), (b) disk instabilities: the star formation in basically all spiral galaxies exhibits patterns which can be linked to the presences of spiral density waves and/or large-scale stellar bars. Stellar bars are quite preponderous (about $75 \%$ of all spirals are classified as barred, e.g. Laine et al. 2002) and are often associated with nuclear starbursts or the presence of so-called starburst rings (e.g. Buta \& Crocker 1993). (c) galaxy mergers: galaxy interactions and mergers are believed to result in tremendous star formation activities and rates observed for example in ultraluminous Infra-Red galaxies (ULIRGs) or some quasi-stellar objects (QSOs).

\section{Star Formation in the Local Universe}

The local universe offers the great advantage to study in detail the impact of different triggering mechanism on the mode of star formation. Numerous studies using high angular resolution data from either ground-based telescopes or the Hubble Space Telescope (HST) have shown that the properties of the stellar clusters, young or old, are rather similar irrespective of their environment (see also table 1). These young clusters are referred to as young massive clusters (YMCs) or sometimes also as super star clusters (SSCs). However, in contrast to the YMCs, SSCs are believed to be the young counterparts of old globular clusters. In the following, the different mechanisms are briefly summarized and illustrated by some characteristic examples.

\subsection{Random}

Dwarf irregular galaxies are prime examples for random or stochastic star formation. They usually only exhibit a small number of active star forming regions which are in general isolated from each other (e.g., NGC 4214: Walter et al. 2000; see Fig. 1). The distribution of star forming sites appears irregular. Despite the low total star formation rates of $\leq 0.4 \mathrm{M}_{\odot} \mathrm{yr}^{-1}$ (Mateo 1998), the star formation event itself can have a strong impact on the low-mass dwarf galaxy. In extreme cases, a central starburst can even lead to mass-loss and/or disruption of the interstellar medium of the dwarf galaxy (e.g., Martin 1998, MacLow \& Ferrara 1999).

\subsection{Disk Instabilities}

The gas disks of spiral galaxies can become instable, thus allowing the formation of sites of enhanced gas density and therefore triggering the formation of stars. The cause for these instabilities can be either instantaneous or due to tidal interactions with neighboring galaxies. In the following the most common disk instabilities are discussed in more detail.

Spiral Density Waves The nearby grand-design spiral galaxy M 51, also known as the Whirlpool galaxy, is a prime example for the presences of spiral density waves (Fig. 2). Its total star formation rate of $\sim 4 \mathrm{M}_{\odot} \mathrm{yr}^{-1}$ (e.g. Scoville et al. 2001 ) is slightly above the average value found for these kind of galaxies.

Detailed studies of the stellar inventory make use of the excellent angular resolution provided by the HST. The central bulge of M51 consists mainly of an old ( $>5$ Gyr) stellar population. In addition to that, Lamers et al. (2002) 


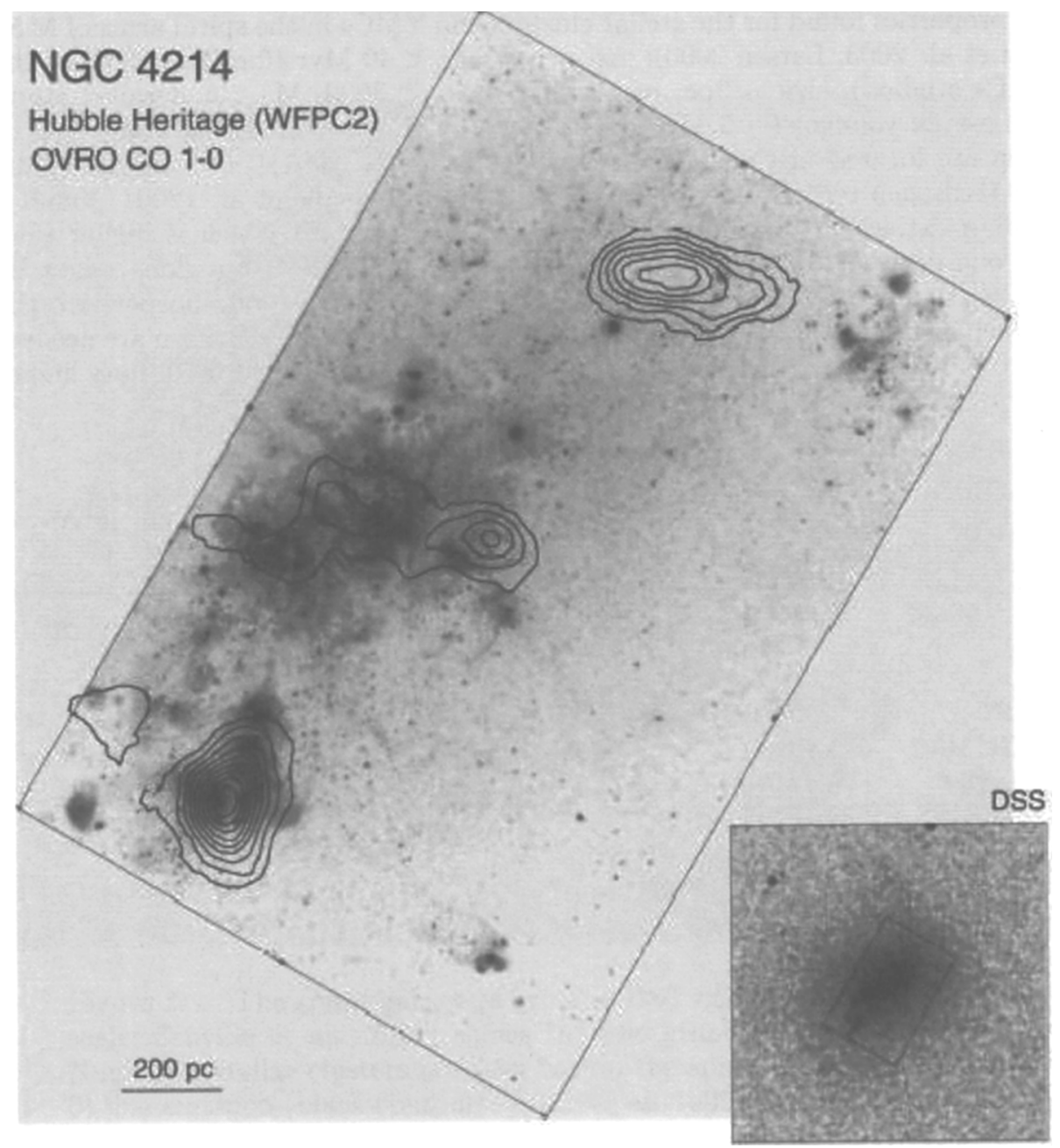

Figure 1. The dwarf galaxy NGC 4214. The intense star formation (large image; gray-scale) is irregularly distributed within the optical body (small inset) of NGC 4214. The contours show the CO line emission as observed with the OVRO $\mathrm{mm}$-interferometer (Walter et al. 2001). 
identified numerous sources representing either single massive stars or low-mass stellar clusters in the bulge. These basically un-obscured clusters have typical ages of 1-20 Myr, and sizes of less than $4 \mathrm{pc}$. The cluster masses are estimated to be $<1000 \mathrm{M}_{\odot}$ from comparison of the colors of the clusters and predictions from population synthesis programs. These numbers are remarkably similar to the properties found for the stellar clusters and YMCs in the spiral arms of M 51 (Bik et al. 2003, Larsen 2000): $A_{V} \sim 1-3$, age $<40 \mathrm{Myr}$ (for about $60 \%$ of the YMCs studied), size $\sim 3 \mathrm{pc}$, and stellar mass $<3000 \mathrm{M}_{\odot}$. A detailed study of the even younger ( $\sim 5 \mathrm{Myr}) \mathrm{HII}$ regions in M 51 revealed that about half of them are located in the spiral arms (Scoville et al. 2001). By comparing the two Hydrogen recombination lines $\mathrm{H} \alpha$ and $\mathrm{Pa} \alpha$, Scoville et al. (2001) found a median extinction towards the HII regions of $\mathrm{A}_{V} \approx 3.1$ which is higher than the one derived for the YMCs. This suggests that the HII regions might be still surrounded by some parent molecular material. To probe deeper into the molecular material other tracers which are less effected by extinction are needed, since the average extinction within the gas spiral arms is about 5-10 times higher than the one derived for the HII regions (Schinnerer et al. in prep.).

Table 1. Properties of Stellar Clusters in Various Environments

\begin{tabular}{lrrrrl}
\hline \hline Galaxy & $\begin{array}{r}A_{V} \\
{[\mathrm{mag}]}\end{array}$ & $\begin{array}{r}\text { Age } \\
{[\mathrm{Myr}]}\end{array}$ & $\begin{array}{r}\text { Size } \\
{[\mathrm{pc}]}\end{array}$ & $\begin{array}{r}\text { Mass } \\
{\left[\mathrm{M}_{\odot}\right]}\end{array}$ & Ref. \\
\hline M 51 & $<1$ & $1-20$ & $<4$ & $<10^{3}$ & Bulge $^{1}$ \\
& $\sim 3.1$ & $\sim 5$ & $\sim 30$ & $<5 \times 10^{3}$ & HII reg. $^{2}$ \\
& $\sim 1-3$ & $<40$ & $\sim 3$ & $<5 \times 10^{3}$ & YMC $^{3,4}$ \\
M 82 & $5-25$ & $<15-600$ & $5-20$ & $<5 \times 10^{3}$ & Nuc. starburst $^{5,6}$ \\
NGC 1512 & $<1-\gg 1$ & $1-100$ & $<5$ & $<5 \times 10^{3}$ & Starburst Ring $^{7}$ \\
Arp 244 & $1-6 \rightarrow 70$ & $5-10 ; \sim 100$ & $\sim 4-30$ & $0.7-5 \times 10^{6}$ & Merger $^{8,9,10}$ \\
\hline \hline
\end{tabular}

1: Lamers et al. (2002),

2: Scoville et al. (2001),

3: Larsen (2000),

4: Bik et al. (2003),

5: de Grijs et al. (2001),

6: de Grijs, et al. (2003),

7: Maoz et al. (2001),

8: Mengel et al. (2001),

9: Gilbert et al. (2000),

10: Whitmore et al. (1999)

Nuclear Starbursts The almost edge-on galaxy M 82 is also known as one of the proto-typical starburst galaxies with a total star formation rate of $\sim 10 \mathrm{M}_{\odot} \mathrm{yr}^{-1}$. The central starburst is energetic enough to drive an enormous outflow of ionized gas (e.g. Devine \& Bally 1999) and molecular gas (Walter et al. 2002). The nuclear stellar population has been studied in detail by de Grisj et al. (2001, 2003). Due to its edge-on orientation in conjunction with the massive nuclear star formation the average extinction in the center of M 82 is in the range of $5-25$. Besides a population of young $(<15 \mathrm{Myr})$ stellar clusters, an older (fossil) starburst population is present in the nuclear region (de Grijs et al. 2001, 2003). 


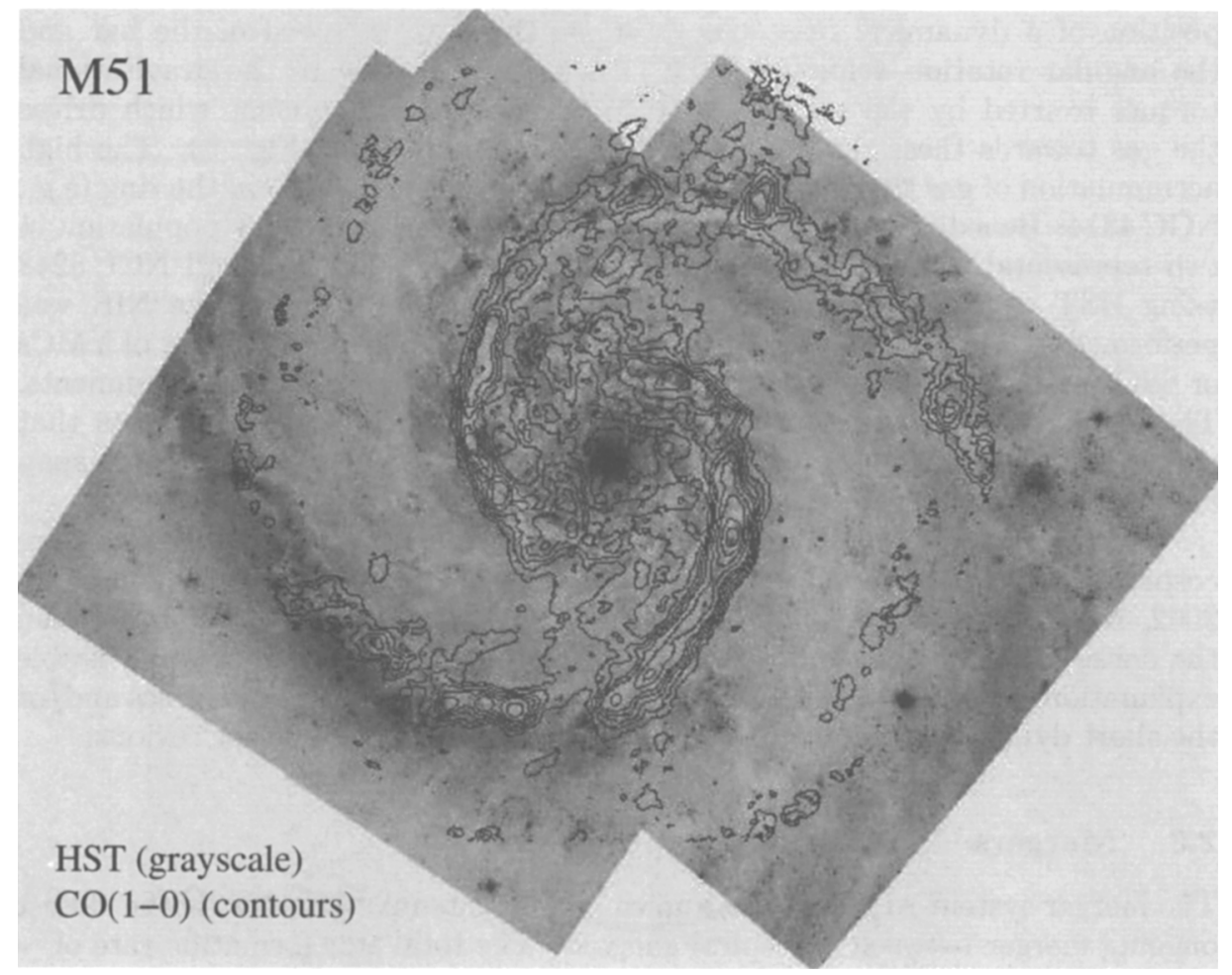

Figure 2. The spiral galaxy M 51. The HST composite image (grayscale; Scoville et al. 2001) shows the two grand-design spiral arms. Numerous stellar clusters are seen within the spiral arms. The $\mathrm{CO}(1-$ 0 ) line emission (black contours; Aalto et al. 1999) roughly follows the optical spiral arms. Estimates of the $\mathrm{H}_{2}$ density suggest that the extinction in the molecular spiral arms is about 5-10 times higher than the one derived for the HII regions using optical and NIR $\mathrm{H}$ recombination lines (Schinnerer et al. in prep.). 
The stellar clusters of this $\sim 600$ Myr old component have sizes of 5-20 pc and masses of $\sim 10^{5} \mathrm{M}_{\odot}$ similar to those of YMCs seen for example in M 51 .

Starburst Rings Circumnuclear star formation or starburst rings are commonly observed in barred spiral galaxies. These nuclear rings have typical diameter of 1-2 kpc (Buta \& Crocker 1993). Their formation is very likely intimately linked to the presence of large-scale stellar bars. The rings are usually located at the position of a dynamical resonance between the pattern speed of the bar and the angular rotation velocity of the galaxy. This is due to the gravitational torques exerted by the stellar bar onto the gaseous component which drives the gas towards these resonances (e.g., Athanassoula 1992, Fig. 3). The high accumulation of gas then might allow for massive star formation in the ring (e.g., NGC 4314: Benedict et al. 1996). Detailed analysis of the stellar population in two representative circumnuclear starburst rings in NGC 1512 and NGC 5248 using HST continuum and emission line data from the UV to the NIR was performed by Maoz et al. (2001). Both galaxies harbor a large number of YMCs or SSCs with sizes and masses similar to YMCs observed in other environments. The comparison between the location of SSCs and HII regions suggests that young star forming clusters can only be obscured for a very short-time span before they become visible as HII regions.

However, not all starburst rings show that the sites of star formation are cospatial with the reservoir of molecular gas (e.g. NGC 4303: Schinnerer et al. 2002, NGC 5248: Jogee et al. 2002). This suggests that the relation between the dense molecular gas and the actual formed stars is more complex. Possible explanations for the non-correlation might be related to the gas dynamics and/or the short dynamical timescales of a few 10-100 Myr in the nuclear regions.

\subsection{Mergers: Production of Star Clusters}

The merger system Arp 244 (also known as the Antennae galaxies) is the closest ongoing merger between two spiral galaxies. The total star formation rate of $20 \mathrm{M}_{\odot} \mathrm{yr}^{-1}$ is at the lower end observed in the high-luminosity ULIRGs such as Arp 220 (star formation rate of $\sim 300 \mathrm{M}_{\odot} \mathrm{yr}^{-1}$ ). HST observations revealed at least 800 resolved star clusters, with up to 8000 possible (unresolved) candidates (Whitmore et al. 1999). The extinction rises from a few magnitudes to up to 70 in the overlap region (Mirabel et al. 1998), making the study of clusters in that region extremely difficult. Mid-IR observations with ISO showed that $15 \%$ of the total MIR luminosity is arising from a distinct position in the overlap region (Mirabel et al. 1998). However, due to the lack of spatial resolution no assessment of these possibly youngest sites of star formation was possible. The typical derived masses and sizes of the (optical) stellar clusters (Whitmore et al. 1999) are similar to those found in other environments, but can be an order of magnitude higher for the largest identified cluster. The stellar clusters can be divided into three age ranges of (a) $<5$ Myrs, mainly found at the edge of the overlap region, (b) 5-10 Myr mainly in the western loop, and (c) about $100 \mathrm{Myr}$ in the northeastern star forming region (Whitmore et al. 1999). The similar physical properties of the stellar clusters are not totally expected, since the observed giant molecular cloud associations (GMAs) in the antennae have 


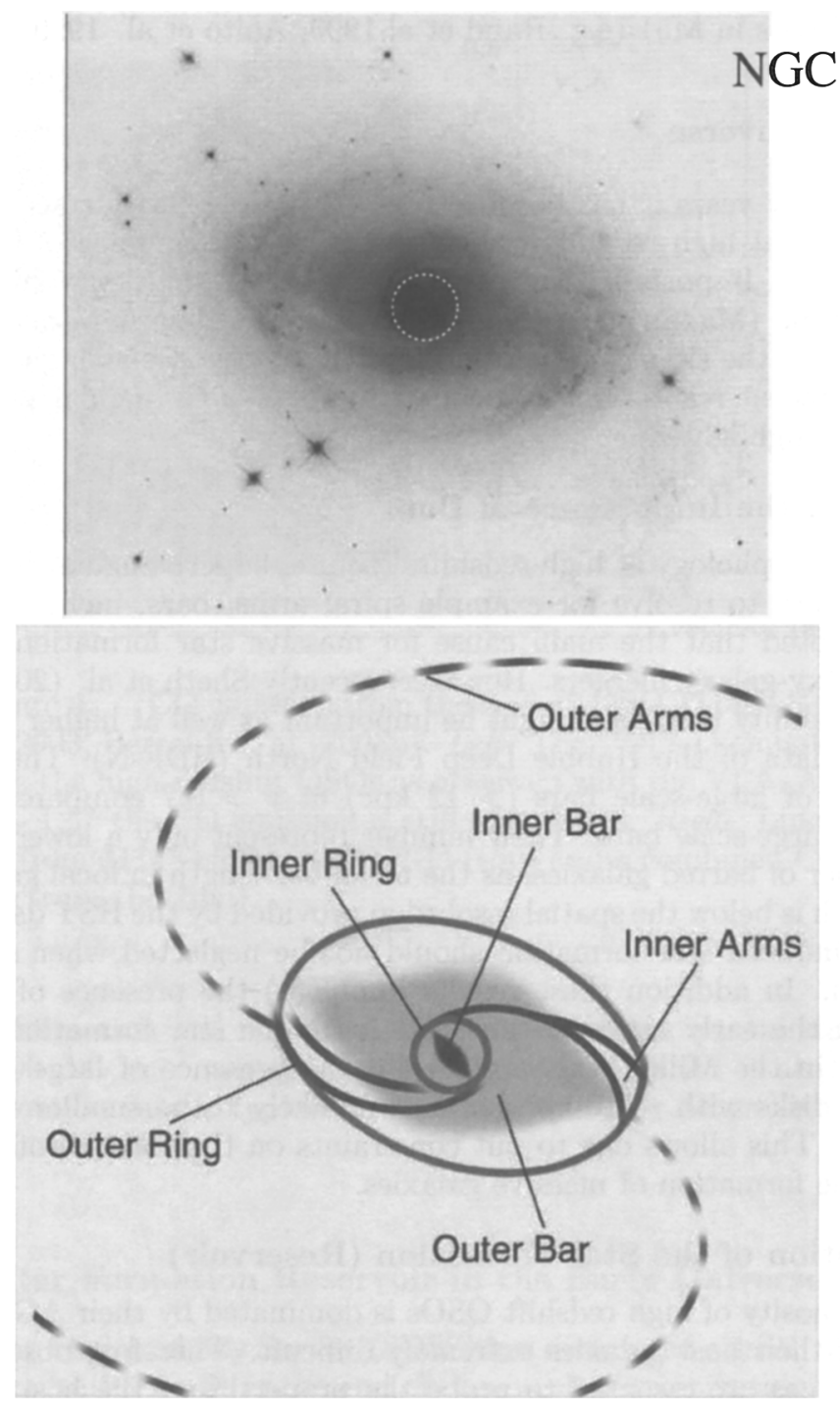

Figure 3. The circumnuclear starburst ring of NGC 6782 (Windhurst et al. 2002, HST Heritage image). Top: Composite color HST image showing the prominent dust lanes along the bar. The UV starburst ring is indicated by the white dashed circle. Bottom: Sketch of the various components present in NGC 6782 (courtesy of Frattare, STScI). The starburst ring is located inside the large-scale bar which is thought to induce gas infall toward the ring along the dust lanes. 
molecular gas masses of 3-6 × $10^{8} \quad \mathrm{M}_{\odot}$ (Wilson et al. 1999) about 5-10 more massive as the GMAs in M51 (e.g. Rand et al.1993, Aalto et al. 1999).

\section{The high-z universe}

Only in the past few years it has become possible to detect and trace the star formation reservoir at high redshift when the universe had an age of less than half its current one. Important issues besides the star formation volume density at a given time (Madau plot) include the triggering mechanisms for star formation as well as the sites of star formation within galaxies at high redshift. In the following recent results in this field (relevant to high angular resolution observations) are highlighted.

\subsection{Revisiting the Importance of Bars}

Studying galaxy morphology at high redshifts requires superb angular resolution and imaging quality to resolve for example spiral arms, bars, mergers etc. It is generally accepted that the main cause for massive star formation at high redshifts are galaxy-galaxy mergers. However, recently Sheth et al. (2003) have revisited the possibility that bars might be important as well at higher redshifts using NICMOS data of the Hubble Deep Field North (HDF-N). They found a preponderance of large-scale bars $(>12 \mathrm{kpc}$ ) at $z>0.7$ compared to the local fraction of large-scale bars. Their number represent only a lower limit to the actual number of barred galaxies, as the mean bar length in local galaxies is about $5 \mathrm{kpc}$ which is below the spatial resolution provided by the HST data. This shows that bar-induced star formation should not be neglected when studying galaxy evolution. In addition these results imply (a) the presence of massive galactic disks in the early universe consistent with the star formation history of the thin disk in the Milky Way, and/or (b) the presence of large disks, as merger induced disks with $\sim 10$-kpc bars are unlikely to be smaller than the bars themselves. This allows one to put constraints on the theories of galaxies evolution and the formation of massive galaxies.

\subsection{Distribution of the Star Formation (Reservoir)}

The optical luminosity of high redshift QSOs is dominated by their AGNs making the study of their host galaxies extremely difficult. Therefore observations of the molecular gas are essential to probe the properties of the host galaxies themselves. In general it is not possible yet to resolve the distribution of the molecular gas in highest redshift QSOs. However, the different spatial distribution of the various components (central AGN, stellar disk+bulge, gas disk) can be studied in lensed QSOs, as these components might have different lensed images depending on the exact geometry as well as on the properties of the lens itself. The lensed QSO J2322+1922 at a redshift of $\mathrm{z}=4.12$ is to-date the highest redshift source for which a size of its star forming region could be derived. Modeling the different appearances of the optical quasar light and the distribution of the molecular gas in its $\mathrm{CO}(2-1)$ line with a lensed model gives a size of $4 \mathrm{kpc}$ for the molecular gas disk (Carilli et al. 2003). The derived molecular gas reservoir is quite substantial with $\mathrm{M}\left(\mathrm{H}_{2}\right) \sim 2 \times 10^{11} \mathrm{M}_{\odot}$. The coincidence of the emission of molecular gas and radio continuum implies that the radio emission is powered 
$\mathrm{J} 1148+5251(\mathrm{z}=6.42)$
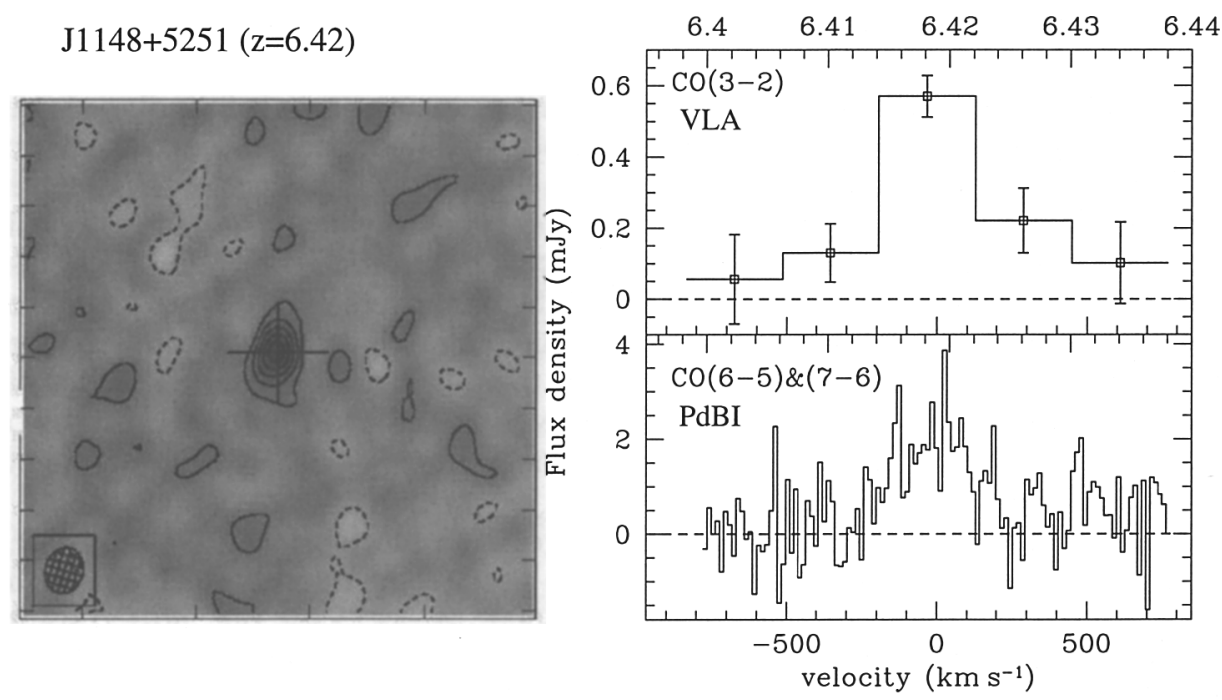

Figure 4. $\quad \mathrm{CO}$ emission from the $\mathrm{z}=6.42 \mathrm{QSO} \mathrm{J} 1148+5251$ (Walter et al. 2003, Bertoldi et al. 2003b). Left: The $\mathrm{CO}(2-1)$ emission associated with the high-redshift QSOs as observed with the VLA. At a resolution of $\sim 1.5$ " the CO emission is still unresolved. Right: Line spectra arising from $\mathrm{J} 1148+5251$ in $\mathrm{CO}(2-1)$ (top) and a combined $\mathrm{CO}(6-5) \&(7-6)$ spectrum (bottom).

by star formation and not the AGN. This results in a total star formation rate of $\sim 3000 \mathrm{M}_{\odot} \mathrm{yr}^{-1}$ using the radio luminosity (Carilli et al. 2003). This high star formation rate is typical to the ones found for other high redshift quasars (Carilli et al. 2001).

\subsection{Star Formation Reservoir in the Early Universe}

The Sloan Digitized Sky Survey (SDSS) has discovered a small number of quasars at high redshift $(z>6)$ at the end of the epoch of reionization when the universe had only an age of $<1 \mathrm{Gyr}$ (Fan et al. 2003). The most distant QSO known to-date lies at a redshift of $\mathrm{z}=6.42$. J1148 +5251 has not only been detected in its dust continuum at $1.2 \mathrm{~mm}$ (Bertoldi et al. 2003a), but also in its CO line emission using both the VLA and the IRAM interferometer (Fig. 4; Walter et al. 2003, Bertoldi et al. 2003b). The derived dust and molecular gas masses of $\mathrm{M}_{\text {dust }} \sim 7 \times 10^{8} \quad \mathrm{M}_{\odot}$ and $\mathrm{M}\left(\mathrm{H}_{2}\right) \sim 2 \times 10^{10} \quad \mathrm{M}_{\odot}$ are quite massive given the fact that the timescale for metal enrichment via massive star formation is fairly short (few $100 \mathrm{Myr}$ ). The VLA limit the size of the CO disk to the range 1 $\mathrm{kpc}<$ diameter $<8 \mathrm{kpc}$ similar to present day galaxies. Clearly, similar studies of more high redshift sources are essential to expand our understanding of star formation in the early universe. It is expected that future surveys will detect more sources well beyond $z>6$. 


\section{Summary and Conclusion}

Tremendous progress has been made in the last years in our understanding of the star formation processes in the local universe. Detailed studies of extragalactic stellar clusters (and populations) in various dynamically distinct environments from nuclear starburst over spiral arms to mergers has shown that the physical properties (size, mass) of the newly formed stellar clusters are very similar. This suggest that the star formation process might be universal even when going to very high redshifts. While the general large-scale triggering mechanisms are understood, the detailed interplay between the gas dynamics and the onset of star formation is still unclear at best. The preferred mode of star formation observed in external galaxies is the formation of clusters with typical masses of a few $1000 \mathrm{M}_{\odot}$.

Mergers are thought to be the main trigger of star formation at high redshift, however the role of bar-driven star formation needs to be re-evaluated. The high estimated total star formation rates of $\sim 3000 \mathrm{M}_{\odot} \mathrm{yr}^{-1}$ for the most distant QSOs are extremely high, and need further investigation with larger samples which should be assembled in the near future. New observatories such as JWST, SIRTF, ALMA, EVLA/SKA, which will be available within the next decade will be the key to probe the obscured star formation locally and at high redshifts with high angular resolution and sensitivity.

\section{References}

Aalto, A., Hüttemeister, S., Scoville, N.Z., Thaddeus, P., 1999, ApJ, 522, 165

Benedict, G.F., Smith, B.J., Kenney, J.D.P., 1996, AJ, 111, 1861

Bertoldi, F., Carilli, C.L., Cox, P., Fan, X., Strauss, M.A., Beelen, A., Omont, A., Zylka, 2003a, A\&A, 406, L55

Bertoldi, F., Cox, P., Neri, R., Carilli, C.L., Walter, F., Omont, A., Beelen, A., Henkel, C., Fan, X., Strauss, M.A., Menten, K.M., 2003b, astro$\mathrm{ph} / 0307408$

Bik, A., Lamers, H.J.G.L.M., Bastian, N., Panagia, N., Romaniello, M., 2003, A\&A, 397, 473

Buta, R., Crocker, D.A., 1993, AJ, 105, 1344

Carilli, C. L., Bertoldi, F., Rupen, M. P., Fan, X., Strauss, M.A., Menten, K. M., Kreysa, E., Schneider, Donald P., Bertarini, A., Yun, M. S., Zylka, R., 2001, ApJ, 555, 625

Carilli, C.L., Lewis, G.F., Djogovski, S.G., Mahabal, A., Cox, P., Bertoldi, F., Omont, A., 2003, Science, Vol. 300, Issue 5620, 773

Devine, D., Bally, J. 1999, ApJ, 510, 197

Fan, X., Strauss, M., Schneider, D., et al., 2003, AJ, 125, 1649

Gilbert, A.M., Graham, J.R., McLean, I.S., Becklin, E.E., Figer, D.F., Larkin, J.E., Levenson, N.A., Teplitz, H.I., Wilcox, M.K., 2000, ApJ, 533, L57

de Grijs, R., O'Connell, R.W., Gallagher III, J.S., 2001, AJ, 121, 768

de Grijs, R., Bastian, N., Lamers, H.J.G.L.M., 2003, MNRAS, 340, 197 
Jogee, S., Shlosman, I., Laine, s., Englmaier, P., Knapen, J.H., Scoville, N.Z., Wilson, C., 2002, ApJ, 575, 156

Laine, S., Shlosman, I., Knapen, J.H., Peletier, R.F., 2002, ApJ, 567, 97

Lamers, H.J.G.L.M., Panagia, N., Scuderi, S., Romaniello, M., Spaans, M., de Wit, W.J., Kirshner, R., 2002, ApJ, 566, 818

Larsen, S.S., 2000, MNRAS, 319, 893

MacLow, M.-M., Ferrara, A., 1999, ApJ, 513, 142

Maoz, D., Barth, A.J., Ho, L.C., Sternberg, A., Filippenko, A.V., 2001, AJ, 121, 3048

Martin, C.L., 1998, ApJ, 506, 222

Mateo, M.L., 1998, ARA\&A. 36, 435

Mengel, S., Lehnert, M.D., Thatte, N., Tacconi-Garman, L.E., Genzel, R., 2001, ApJ, 550, 280

Mirabel, I.F., Vogroux, L., Charmandaris, V., Sauvage, M., Gallais, P. Tran, P., Cesarsky, C., Madden, S.C., Duc, P.A., 1998, A\&A, 333, L1

Rand, R., 1993, ApJ, 404, 593

Schinnerer, E., Maciejewski, W., Scoville, N.Z., Moustakas, L.A., 2002, ApJ, 575,826

Scoville,N.Z., Polletta, M., Ewald, S., Stolovy, S.R., Thompson, R.M., Rieke, M., 2001, AJ, 122, 3017

Sheth, K., Regan, M.W., Scoville, N.Z., Strubbe, L.E., 2003, ApJ, 592, L13

Walter, F., Bertoldi, F., Carilli, C., cox, P., Lo, K.Y., Neri, R., Fan, X., Omont, A., strauss, M.A., Menten, K.M., 2003, Nature, 424, 406

Walter, F., Taylor, C.L., Hüttemeister, S., Scoville, N.Z. McIntyre, V., 2001, AJ, 121, 727

Walter, F., Weiss, A., Scoville, N.Z., 2002, ApJ, 580, L21

Windhorst, R.A., Taylor, V.A., Jansen, R.A., et al., 2002, ApJS, 143, 113

Whitmore, B.C., Zhang, Q., Leitherer, C., Fall, S.M., Schweizer, F., Miller, B.W., 1999, AJ, 118, 1551

Wilson, C., Scoville, N., Madden, S.C., Charmandaris, V., 2000, ApJ, 542, 120 